\title{
Astérisque
}

\section{S. BAOUENDI}

\section{Solvability of partial differential equations in the traces of analytic solutions of the heat equation}

\author{
Astérisque, tome 2-3 (1973), p. 86-97
}

<http://www.numdam.org/item?id=AST_1973_2-3_86_0>

(C) Société mathématique de France, 1973, tous droits réservés.

L'accès aux archives de la collection « Astérisque » (http://smf4.emath.fr/ Publications/Asterisque/) implique l'accord avec les conditions générales d'utilisation (http://www.numdam.org/conditions). Toute utilisation commerciale ou impression systématique est constitutive d'une infraction pénale. Toute copie ou impression de ce fichier doit contenir la présente mention de copyright.

\section{NumDam}

Article numérisé dans le cadre du programme Numérisation de documents anciens mathématiques http://www.numdam.org/ 
SOLVABILITY OF PARTIAL DIFTERENTIAL EQUATIONS

IN THE TRACES OF ANALYTIC SOLUTIONS OF THE HEAT EQUATION

by

M.S. BAOUENDI

Purdue University

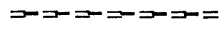

N. ARONSZAJN introduced in his lecture at this colloqiurn [1] an abstract FRECHET space $C H$ : "the traces" of the analytic solutions of the heat equation. In this talk, we give additional properties and discuss the solvability of partial differential equations in $\mathrm{CHb}$. As examples, we prove the solvability in this space of some first order operator's which are solvable neither in the space of distributions, nor in the space of SATO-MARTINEAU hyperfunctions .

The complete procfs will be published elsewhere([2]).

\section{I- Définitions, notations and basic properties}

We denote by $\mathbb{R}^{n}$ (resp. $\mathbb{C}^{n}$ ) the $n$ dimensional real (resp.complex) space. We introduce the following notations:

$$
\begin{gathered}
\mathbb{a}_{+}=\left\{x \in \mathbb{d}^{1}, \text { Re } x>0\right\}, \overline{\mathbb{c}}_{+}=\left\{x \in \mathbb{d}^{1}, \text { Re } x \geqslant 0\right\} \\
\mathbb{a}_{+}^{n}=\mathbb{d}^{n-1} \times \mathbb{c}_{+}, \quad \overline{\mathbb{d}}_{+}^{n}=\mathbb{a}^{n-1} \times \overline{\mathbb{d}}_{+} \cdot \\
-86-
\end{gathered}
$$




$$
\text { If } x \in \mathbb{0}^{n} \quad, x=\left(x_{1}, \ldots . x_{n}\right)=\left(x^{1}, x_{n}\right)
$$

with

$$
\begin{aligned}
& x^{\prime}=\left(x_{1}, \ldots \ldots x_{n-1}\right), \\
& x^{2}=\sum_{i=1}^{n} x_{i}^{2} \quad|x|^{2}=\sum_{i=1}^{n}\left|x_{i}\right|^{2}
\end{aligned}
$$

where $\left|x_{i}\right|$ denotes the modulus of the complex number $x_{i}$. If $\alpha$ is a multiindex, $\alpha=\left(\alpha_{1} \ldots \ldots \alpha_{n}\right), \alpha_{i}$ integer $>0$ we denote

$$
D_{x}^{\alpha}=D^{\alpha}=\frac{\partial^{\alpha_{1}}}{\partial x^{\alpha_{1}}} \cdots \cdots \frac{\partial^{\alpha_{n}}}{\partial x_{n}^{\alpha_{n}}}, x \in \mathbb{C}^{n} \text { or } x \in \mathbb{R}^{n} \text {. }
$$

If $\Omega$ is an open set in $e^{n}$, we denote by $H(\Omega)$ the space of analytic functions defined in $\Omega$, with the usual topology ; and $H^{\prime}(\Omega)$ its dual, the space of analytic functionals in 8 .

We denote

$$
E(x, t)=\frac{1}{(4 \pi t)^{\frac{n}{2}}} \exp \left(-\frac{x^{2}}{4 t}\right),(x, t) \in \mathbb{x}_{n}^{n+1}
$$

Letcth $=c \mathbb{H}_{n}$ be the space of analytic solutions $u$ of the heat equation

$$
-\frac{\partial u}{\partial t}-\sum_{i=1}^{n}-\frac{\partial^{2} u}{\partial x_{I}^{2}}=0
$$

defined in $\mathbb{a}_{+}^{n+1}+\mathbb{H}$ is a closedsubspace of $\mathrm{H}\left(\mathbb{a}_{+}^{\mathrm{n}+1}\right)$.

The mapping

$$
\begin{aligned}
& c h_{h_{n}}\left(H\left(c_{+}^{n}\right)\right)^{2} \\
& u \mapsto\left(u_{0}, u_{1}\right)
\end{aligned}
$$

where $u_{0}$ and $u_{\uparrow}$ are the CAUCHY data defined by

$$
\begin{aligned}
& u_{0}\left(x^{\prime}, t\right)=u\left(x^{\prime}, 0, t\right) \\
& u_{1}\left(x^{\prime} t\right)=-\frac{\partial u}{\partial x_{n}}\left(x^{\prime}, 0, t\right),
\end{aligned}
$$

is a topological isomorphism. (The inverse mapping is given by the solution of the 
corresponding global CAUCHY problem).

If $f$ is, say a tempered distribution defined on $1 \mathrm{R}^{\mathrm{n}}$, we denote

$$
\tilde{f}(x, t)=\langle f(y), E(x-y, t)\rangle
$$

In fact, (I.3) defines a topological embedding of $\mathscr{S}\left(\mathbb{R}^{n}\right), H^{\prime}\left(\mathbb{C}^{n}\right)$ into cttb $n$ with dense range. Therefore, we have a natural topological embedding of the dual cthb; into $\mathscr{S}\left(\mathbb{R}^{n}\right)_{n H}\left(\mathbb{C}^{n}\right)$. We will give in the next section a com-

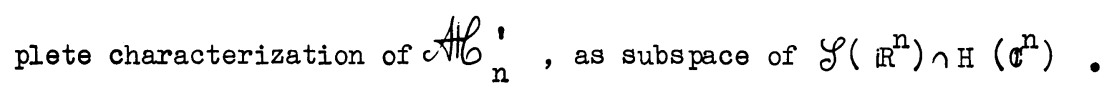

From now on, we condiderctil as an abstract space which contains $y^{\prime}\left(\mathbb{R}^{n}\right), H^{\prime}\left(\mathbb{e}^{n}\right)$. etc... . If u EcHb, we denote

$$
\tilde{u}(x, t)
$$

the value of the corresponding solution of the heat equation at $(x, t) \in \mathbb{c}_{+}^{n+1}$. An element incth is called a "trace".

We refer to [1] where the space c Hb is introduced and where other properties are discussed.

II. Characterization of the dual space, the multipliers and the convolutors

We considerctll ${ }_{n}$ as a subspace of ct b $b_{n}$. We have the following

PROPOSITION II.1.-

$(I I .1)$

1)- A trace $u$ is inut ${ }_{n}^{\prime}$ if and only if there exists $F \in H^{\prime}\left(a_{+}^{n+1}\right)$ (non-unique) such that, for $(x, t) \in \mathbb{c}_{+}^{n+1}$

$$
\tilde{u}(x, t)=\langle F(x, \tau), \quad E(x-y, t+\tau)\rangle .
$$

2)- For any $u \in C H H_{n}^{\prime}$, there exists a unique pair $\left(\mathrm{F}_{0}, \mathrm{~F}_{1}\right) \in\left[\mathrm{H} \cdot\left(\mathbb{C}_{+}^{n}\right)\right]^{2}$ such that, for $(x, t) \in \mathbb{C}_{+}^{n+1}$.

(II.2) $\quad \tilde{u}(x, t)=\left\langle F_{0}\left(y^{\prime}, \tau\right) \otimes \delta\left(y_{n}\right)+F_{1}\left(y^{\prime}, \tau\right) \otimes \delta_{0}^{2}\left(y_{n}\right), E(x-y, t+\tau)\right\rangle$ 
SOLVABILITY

where $\delta$ is the DIRAC measure in one variable, and $\delta^{\prime}$ its derivative.

Part 2) follows, in particular from the isomorphism (1.2).

THEOREM II.1.-

Let $f$ be an entire function defined in $\mathbb{q}^{n}$. $f \in \mathrm{Ct}^{\prime} 6^{\prime}$ if an

only if there exist $C \geqslant 0, \mathbb{M} \geqslant 0, A>B \geqslant 0$ such that for any $x \in \mathbb{C}^{n}$

(II.3)

$$
|f(x)|<\exp \left(M|x|+B\left|x^{2}\right|-A \operatorname{Re} x^{2}\right) .
$$

PROOF $1^{\circ}$ Necessity of (II.3)

From (II.1) we get

$$
|\tilde{u}(x)|=|\langle F(y, \tau), E(x-y, \tau)\rangle| \leqslant C \sup _{(y, \tau) \in K} E(x-y, \tau)
$$

where $C \geqslant 0$ and $K$ is a compact set in $\mathbb{0}_{+}^{n+1}$.

The inequality (II.3) follows easily for $\tilde{u}(x)$.

20) Sufficiency of (II, 3)

If $F$ is an anlytic functional in $\mathbb{C}^{\mathrm{n}}$, let us denote

$$
\stackrel{*}{F}(\zeta)=\langle F(x), \exp (-i x, \zeta)\rangle
$$

its Fourier-BOREL transform. If $f \in$ c $^{\prime} 6^{\prime}$, it follows easily from (II.1) and (II.2) that one can obtain the representations :

$$
f(x)=\stackrel{a}{G}\left(x,-i x^{2}\right) \quad, \quad G \in H^{\prime}\left(\mathbb{C}^{n+1}\right)
$$

or

$$
f(x)=G_{0}^{*}\left(x^{\prime},-i x^{2}\right)+x_{n} \hat{a}_{1}\left(x^{\prime},-i x^{2}\right), G_{0}, G_{1} \in H^{\prime}\left(\mathbb{c}_{+}^{n}\right)
$$

In order to prove the sufficiency of (II.3), we assume that a given entire function $f$ satisfies (II.3). We shall show that $f$ may be written in the form (II.5). The latter result is a consequence of the following lemmas. 


\section{LEIMNA II.1.-}

Let $f$ be an entire function in $\mathrm{n}$ complex variables even with respect

to $x_{n}\left(\right.$ i.e $\left.f\left(x^{\prime}, x_{n}\right)=f\left(x^{\prime},-x_{n}\right)\right)$. There exists a unique entire function $g$ defined in $c^{n}$ such that

$$
f\left(x^{\prime}, x_{n}\right)=g\left(x^{\prime}, x^{2}\right)
$$

In addition, $f$ satisfies (II.3) if an only if there exist $C^{\prime} \geqslant 0$, $\mathrm{II}^{\prime} \geqslant 0, \mathrm{~A}^{\prime}>\mathrm{B}^{\prime} \geqslant 0$ such that for $\mathrm{y} \in \mathbb{\mathbb { C }}^{\mathrm{n}}$ and $\tau \in \mathbb{C}$.

$$
|g(y, \tau)|<C^{\prime} \exp \left(\mathrm{M}^{\prime}|y|+B^{\prime}|\tau|-A^{\prime} \operatorname{Re} \tau\right) 。
$$

We observe here, that the condition (II.6) is equivalent to say that there exists $G \in H^{\prime}\left(\mathbb{C}_{+}^{\mathrm{n}}\right)$ such that

$$
g(y, \tau)=\stackrel{*}{G}(y,-i \tau)
$$

$(\operatorname{see}[5],[6])$.

IEMMA (II.2).-

Let $f$ be an entire function satisfying (II.3), and $P$ a polynomial

in $n$ variables with complex.coefficients. If $-\frac{f}{P}$ is entire tt also satisfies (II.3).

The proof of lemma (II.2) uses the inequality

$$
\left|-\frac{f(x)}{P(x)}\right| \leqslant K \sup _{\zeta \in c^{n}}|f(x+\zeta)|
$$

$(\operatorname{see}[5])$.

Remark II.1.- Using the characterization (II.1) and (II.4), it is readily seen that c $\$ 6^{\prime}$ is closed under the FOURIER transform.

From the characterization (II.3) one can obtain 
SOLVABILITY

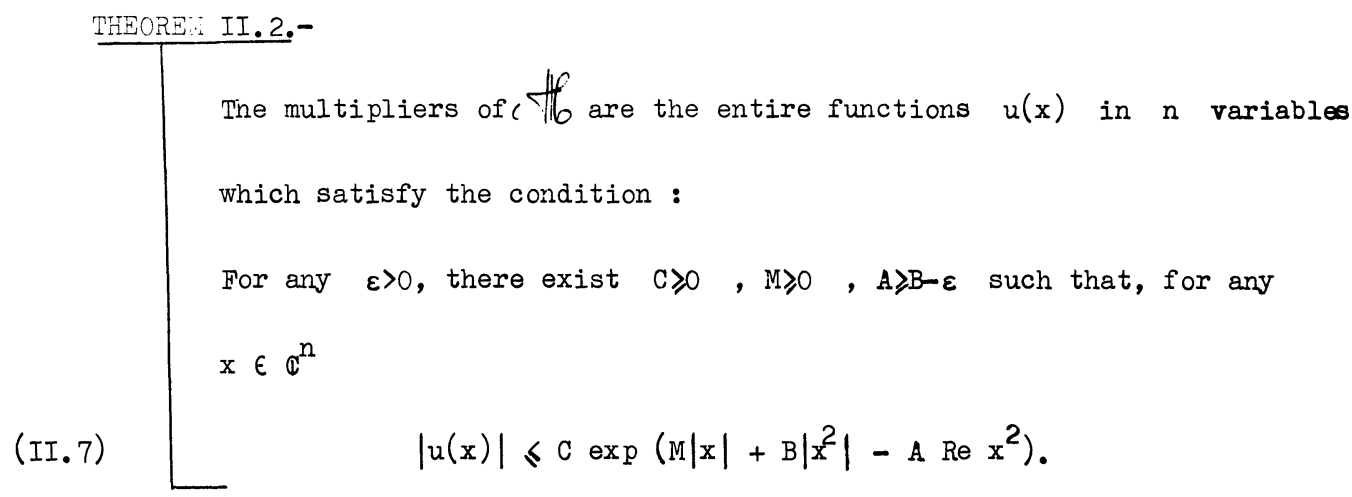

Let us observe in particular, that the space of the multipliers contains the space of entire functions of exponential type.

Let us denote by $\mathscr{b}_{b}$ the space of multipliers, namely the space of entire functions which satisfy (II.7).

The space,$\hat{\mathscr{b}}=\mathcal{G}$ is the space of convolutors. We have in particular the inclusion

$$
H^{\prime}\left(\mathbb{Q}^{n}\right) \subset \mathscr{G}
$$

Let $t_{0}>0$, we denote by $\Sigma_{t_{0}}$ the subspace of $\mathbb{H}_{0}$ defined by :

where $v \in c$ †if.

$$
u \in \Sigma_{t_{0}} \Leftrightarrow \tilde{u}(x, t)=\tilde{v}\left(x, t+t_{0}\right)
$$

The space $\Sigma_{t_{0}}$ is the space of sections at $t_{0} \cdot \Sigma_{t_{0}}$ is provided with the topology of uniform convergence on compact sets of $\mathbb{C}^{\mathrm{n}} \times\{t \in \mathbb{\mathbb { }}, \operatorname{Re} t>-t$ 。 We define the space of sections :

$$
\begin{gathered}
\Sigma={\underset{t}{t_{0} \rightarrow 0}}_{t_{0}>0} \Sigma_{t_{0}} \\
\end{gathered}
$$

We have the following result : 


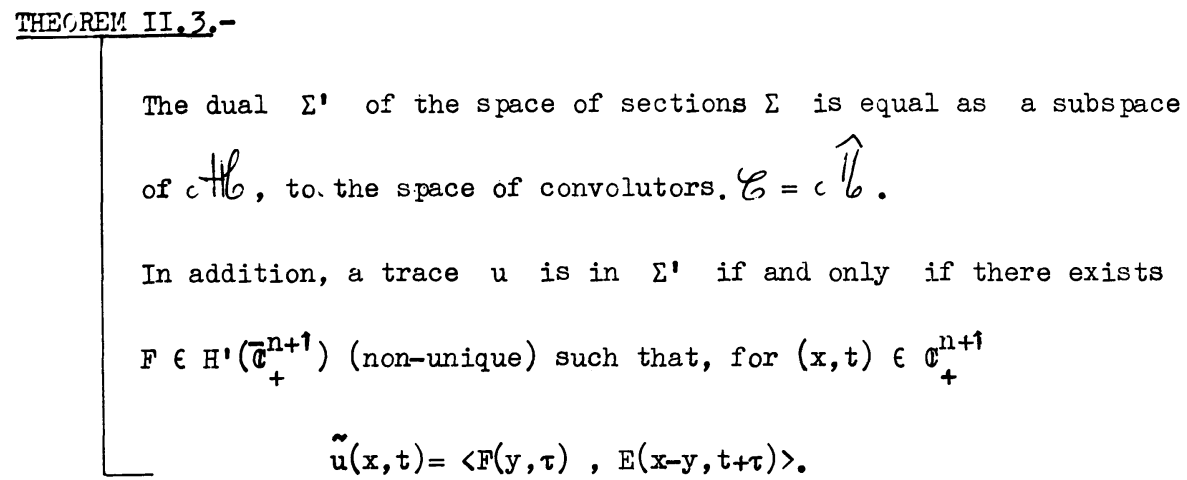

\section{III.- Solvability of P.D.E. with polynomial coefficients}

We consider first, in this section, the constant coefficient case. We prove the possibility of the division by a polynomial inct 6 . Using the Fourier transform, we get the solvability of P.D.E. with constant coefficients, as well as the approximation of the solution of homogeneous equations by exponential-polynomials.

The proofs are based on LEMMA(II.2). In the distribution case, similiar ideas are used in [5].

We have the following results.

\section{THEOREM III.I.-}

Let $\mathrm{P} \neq \mathrm{O}$ be a polynominal in $\mathrm{n}$ variables, with complex coefficients. For any $f \epsilon_{0} \mathbb{T}_{\mathrm{n}}$, there exists

$u \in \mathbb{H}_{\mathrm{n}}$ such that

$$
\mathrm{Pu}=f \text {. }
$$

\section{COROLIAARY III.1.-}

Let $P(D) \neq 0$ be a partial differential operator in $n$ variables with complex coefficients. For any $f \in c t_{b_{n}}$, there exists $u \in c t b_{n}$ such that $\quad P(D) u=f$ 
Let us call exponential-polynomial any entire function $f$ of the form

$$
f(x)=Q(x) \exp (a, x)
$$

where $Q$ is a polynomial and $a \in \mathbb{C}^{n}$. We denote by $V(P(D))$ the space of traces spanned by the exponential-polynomials $f$ satisfying

$$
P(D) f=0
$$

\section{THEOREM III.2.-}

The space $V(P(D))$ is dense in the space

$$
\{u \in c \mathcal{H}, P(D) u=0\}
$$

We shall consider now the polynomial coefficient case. Let $P$ be a differential operator: in $n$ variables $x_{1}, \ldots, x_{n}$ with polynomial coefficients (III.1)

$$
\begin{aligned}
& P=P\left(x, D_{x}\right)=\Sigma a_{\alpha}(x) D^{\alpha} \\
& a_{\alpha} \in C\left[x_{1}, \ldots . x_{n}\right] .
\end{aligned}
$$

We shall discuss the solvability inctlb of the equation

$$
P\left(x, D_{x}\right) u=f
$$

Let us first consider the following operator

$$
\begin{aligned}
& \tilde{P}=\tilde{P}\left(x, t, D_{x}\right)=\sum_{k=0}^{\infty} \frac{t^{k}}{k !} I_{k}\left(x, D_{x}\right) \\
& I_{0}\left(x, D_{x}\right)=P\left(x, D_{x}\right) \\
& I_{k+1}\left(x, D_{x}\right)=\Delta_{x} I_{x}\left(x, D_{x}\right)-I_{k}\left(x, D_{x}\right) \Delta_{x} \\
& \left(\Delta_{x}=\sum_{i=1}^{n} \frac{\partial^{2}}{\partial x_{i}^{2}}\right)
\end{aligned}
$$

$I_{k}$ vanishes for large $k$ and the series (III.3) is in fact a finite sum. The operator $\tilde{P}$ satisfies the following properties :

$$
\tilde{P}\left(x, 0, D_{x}\right)=P\left(x, D_{x}\right)
$$


$\tilde{P}$ commutes with the heat operator $\mathbb{H}=-\frac{\partial}{\partial t}-\dot{L}_{X}$

H $\tilde{\mathrm{P}}=\tilde{\mathrm{P}}$ 且

For any trace $u ; x \in \mathbb{C}^{\mathrm{n}}, \quad t \in \mathbb{C}_{+}$:

$$
(\tilde{P u})(x, t)=\tilde{P}\left(x, t, D_{x}\right) \tilde{u}(x, t) \text {. }
$$

Let us write $\tilde{P}$ in the following form

(III.6)

$$
\tilde{P}\left(x, t, D_{x}\right)=\sum_{k} M_{k}\left(x, t, D_{x^{\prime}}\right) D_{x_{n}}^{2 k}+N_{k}\left(x, t, D_{x^{\prime}}\right) D_{x_{n}}^{2 k+1} \text {. }
$$

where $M_{k}$ and $N_{k}$ are differential operators in $x^{\prime}$, their coefficient being polynomial in $x$ and $t$.

THEOREN III.3.-

The solvability of (III.2) inc Hbis equivalent to the solvability of the following problem :

For any $\left(f_{0}, f_{1}\right) \in\left(H\left(\mathbb{c}_{+}^{n}\right)\right)^{2}$ find

$$
\left(u_{0}, u_{1}\right) \in\left(H\left(\mathbb{e}_{+}^{n}\right)\right)^{2} \text { such that }
$$

(III. 7)

$$
\begin{aligned}
& \sum_{k} M_{k}\left(x^{\prime}, 0, t, D_{x^{\prime}}\right)\left(-\frac{\partial}{\partial t}-\Delta_{x^{\prime}}\right)^{k} u_{0}+N_{k}\left(x^{\prime}, 0, t, D_{x^{\prime}}\right)\left(-\frac{\partial}{\partial t}-\Delta_{x^{\prime}}\right)^{k} u_{1^{\prime}}=f_{0} \\
& \sum_{k}\left(\frac{\partial M_{k}}{\partial x_{n}}\right)\left(x^{\prime}, 0, t, D_{x^{\prime}}\right)\left(-\frac{\partial}{\partial t}-\Delta_{x^{\prime}}\right)^{k_{u_{0}}}+N_{k^{\prime}}\left(x^{\prime}, 0, t, D_{x^{\prime}}\right)\left(-\frac{\partial}{\partial t}-\Delta_{x^{\prime}}\right)^{k+1} v_{v_{0}}+ \\
& \left(\frac{\partial N_{k}}{\partial x_{n}}\right)\left(x^{\prime}, 0, t, D_{x^{\prime}}\right)\left(-\frac{\partial}{\partial t}-\Delta_{x^{\prime}}\right)^{k} u_{1}+M_{k}\left(x^{\prime}, 0, t, D_{x^{\prime}}\right)\left(-\frac{\partial}{\partial t}-\Delta_{x^{\prime}}\right)^{k} u_{1}=f_{1}(*)
\end{aligned}
$$

The isomorphism (I.2) is used in the proof of theorem (III.3). This theorem reduces the solvability of (III.2) incth to the solvability of a system of two P.D.E. with polynomial coefficients in the space of homolorphic functions in $\mathbb{C}_{+}^{n}$, for which global CAUCHY-KOVALEVSKY ty pe theorems may be used.

(*) if $Q(x, D)=\Sigma q_{\alpha}(x) D^{\alpha}$ is a partial differential operator, we denote

$$
\begin{aligned}
\left(\frac{\partial Q_{2}}{\partial \mathrm{x}_{i}}\right)(\mathrm{x}, \mathrm{D})= & \sum \frac{\partial \mathrm{q}_{\alpha}}{\partial \mathrm{x}_{i}}(\mathrm{x}) \mathrm{D}^{\alpha} \\
& -94^{-}
\end{aligned}
$$


Example 1: Let us consider the operator

$$
\frac{\partial}{\partial x_{1}}+i x_{1} \frac{\partial}{\partial x_{2}} \text {. }
$$

It is well known that it is not solvable (even locally) in the space of distributions. $([4],[7], \ldots)$. For the non-solvability in the space of hyperfunctions see [8]. (see also [9]).

\section{THEOREM III.4}

The operator

$$
\begin{gathered}
P=\frac{\partial}{\partial \bar{x}_{1}}+i x_{1}-\frac{u}{\partial x_{2}} \\
\text { is solvable inc } \mathscr{H}_{2} .
\end{gathered}
$$

After a permissible change of variables, the operator $\tilde{P}$ defined in (III.3) becomes in this case

The system (III.7) is of the form

$$
\tilde{\mathrm{P}}=i t\left(-\frac{\partial^{2}}{\partial \mathrm{x}_{1}^{2}}-\frac{\partial^{2}}{\partial \mathrm{x}_{2}^{2}}\right)+\mathrm{P}
$$

(III.8)

$$
\begin{aligned}
& -\frac{\partial^{2} u_{0}}{\partial x_{1}^{2}}=g_{0}+Q_{0}\left(u_{0}, u_{1}\right) \\
& -\frac{\partial^{2} u_{1}}{\partial x_{1}^{2}}=g_{1}+Q_{1}\left(u_{0}, u_{1}\right)
\end{aligned}
$$

where $g_{0}$ and $g_{1}$ are given homolorphic functions in $\mathbb{C}_{+}^{2}$ and $Q_{0}, Q_{1}$ are differential operators of order 1 , acting on $u_{0}$ and $u_{1}$, with respect to the variables $x_{1}$ and $t$, with homolorphic coefficients in $\mathbb{d}_{+}^{2}$. The solvability of (III.8) in $\left(\mathrm{H}_{+}\left(\mathrm{a}_{+}^{2}\right)\right)^{2}$ may be proved using a global CAUCHY-KOVALEVSKY ty pe theorem (see[3] for similar techniques). 


\section{Example 2.-}

THEOREII III.5.-

Let $\alpha$ be a complex number.

The operator

$$
P=x_{2}-\frac{\partial}{\partial x_{1}}+\alpha x_{1}-\frac{\partial}{\partial x_{2}}
$$

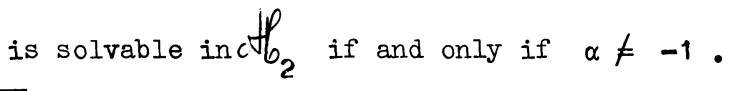

The idea of the proof is similar to that used in theorem (III.4). The non solvability of (III.9) for $\alpha=-1$ was pointed out by R. MOYER.

Remark III.1. - The operator

(III.10)

$$
x_{2}-\frac{\partial}{\partial x_{1}}-x_{1}-\frac{\partial}{\partial x_{2}}
$$

is not solvable in $\mathrm{Alb}_{2}$. However it is possible to prove that, if we consider the "traces of analytic solutions" of the operator

$$
-\frac{\partial}{\partial t}-\frac{\partial^{2}}{\partial x_{1}^{2}}-\lambda-\frac{\partial^{2}}{\partial x_{2}^{2}} \quad ; \quad \lambda>0, \lambda \neq 1
$$

instead of the heat operator, (III.10) is solvable in this new space.

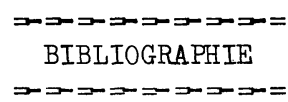

[1] N. ARONSZAJN .- Traces of analytic solutions of the heat operator. This colloqium.-

[2] M.S. BAOUENDI.- Solvability of partial differential equations in the traces of analytic solutions of the heat equation.- Technical report, The University of Kansan, Lawrence, Kansas. U.S.A.- 1972.-

[3] M.S. BAOUENDI and C. GOULAOUIC.- Remarks on the Cauchy problem (to appear)-

[4] I. HORMANDER.- Linear partial differential operators. Springer-Verlag (1963)

[5] B.MALGRANGE.- Existence et approximation des solutions des équations aux dérivées partielles et des équations de convolution. - Ann. Inst. Fourier (1955-56). t.6, p. 271-355. 
[6] A. MARTINEAU.- Sur les fonctionnelles analytiques et la transformée de Fourier-Borel.- Journ. Anal. Math. Jémusalem (1963) t.11,p. 1-164

[7] I. NIRENBERG and F.TREVES.- On local solvability of linear partial differential equations C.P.A.M. (1970), V.23, p 1-38 and V.23 p 459-510.-

[8] P. SCHAPIRA.- Une équation aux dérivées partielles sans solutions dans l'espace des hyperfuntions. C.R. Acad. Sci. (1967), 265, Série A, p.665-667.-

[9] I. SCHWARTZ.- Théorie des distributions , Hermann Paris 1950-1951.-

[10] F. TREVES.- Ovcyannikov theorem and hy perdifferential operators. Notas de Mathematica $N^{\circ} 46$, Istituto de Mathematica Pura e Applicada, Rio de Janeiro, Brazil (1968).- 